\title{
THE TIME DEPENDENCE OF MAGNETIC, VELOCITY, AND INTENSITY FIELDS IN THE SOLAR ATMOSPHERE
}

\author{
NEIL R. SHEELEY, JR.
}

Kitt Peak National Observatory*, Tucson, Ariz. U.S.A.

\begin{abstract}
Spectroheliogram movies of magnetic, velocity, and intensity fields have been obtained in a wide variety of spectral lines. The time resolution was typically 10-30 s. Some of the lines used were: CN $\lambda$ 3883; CaI $\lambda$ 6103; Fer $\lambda$ 4071; Fer $\lambda$ 5434; FeII $\lambda$ 4924; Ca II $\lambda$ 3934; Ca II $\lambda 8542$.

During the summer of 1970 , spectroheliogram movies were made daily using the $38 \mathrm{~cm}$ solar image provided by the east auxiliary telescope at the Kitt Peak National Observatory. The use of a very fast film (Kodak 2485) permitted rapid scanning of the spectroheliograph resulting in a time resolution ranging from $10 \mathrm{~s}$ to $30 \mathrm{~s}$ depending
\end{abstract}

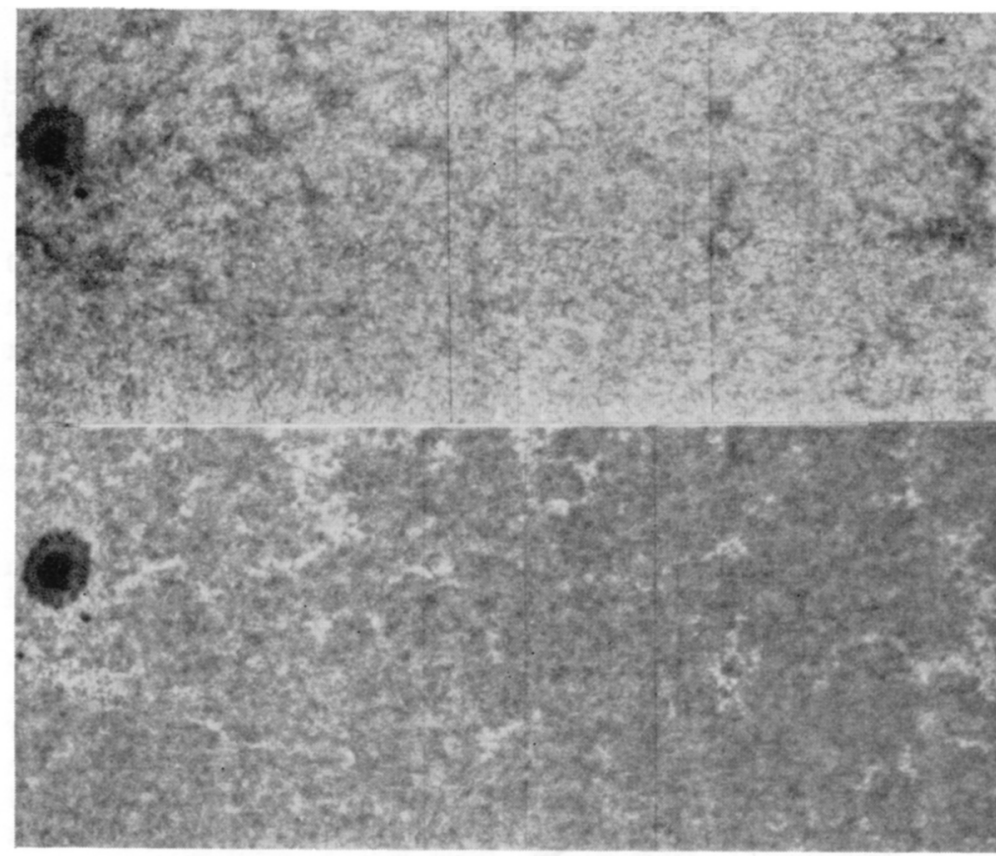

$\mathrm{Fe}$ II

$4924 R$

\section{JULY 12, 1970}

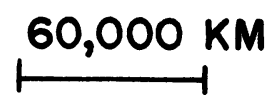

Fig. 1. Comparison between the dark network in FeII $\lambda 4924$ and the bright network in FeI $\lambda 4071$.

* Operated by the Association of Universities for Research in Astronomy Inc., under contract with the National Science Foundation. 


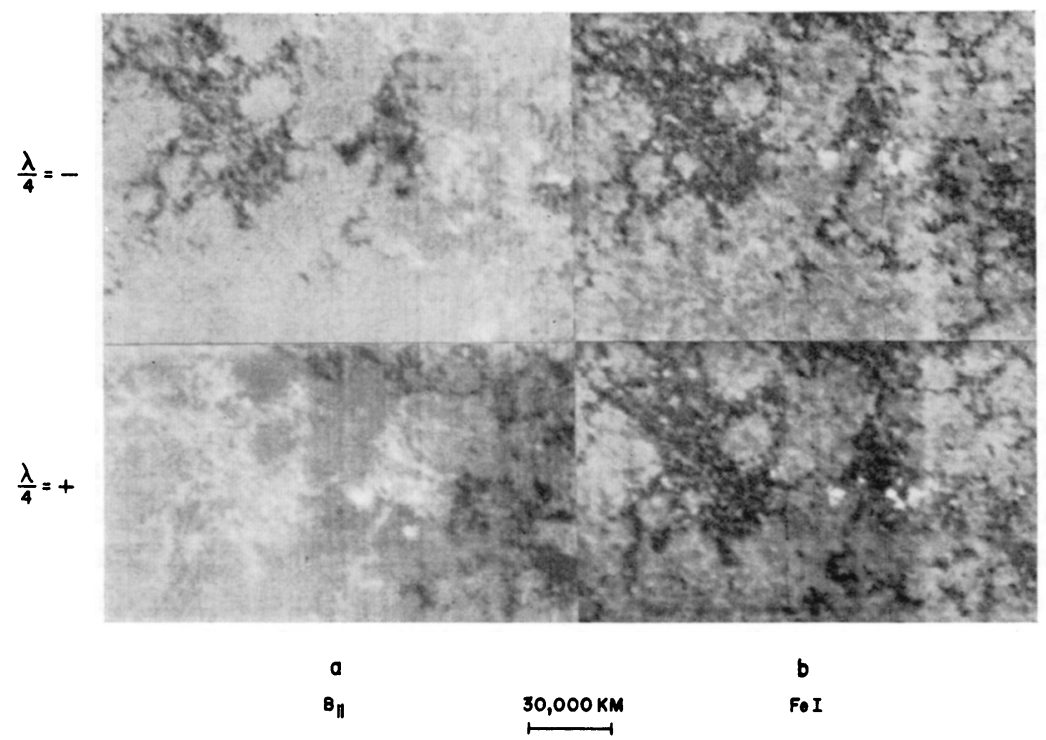

Fig. 2. Comparison between the bright network in FeI $\lambda 4071$ and the magnetic field distribution obtained using CaI $\lambda 6103$.

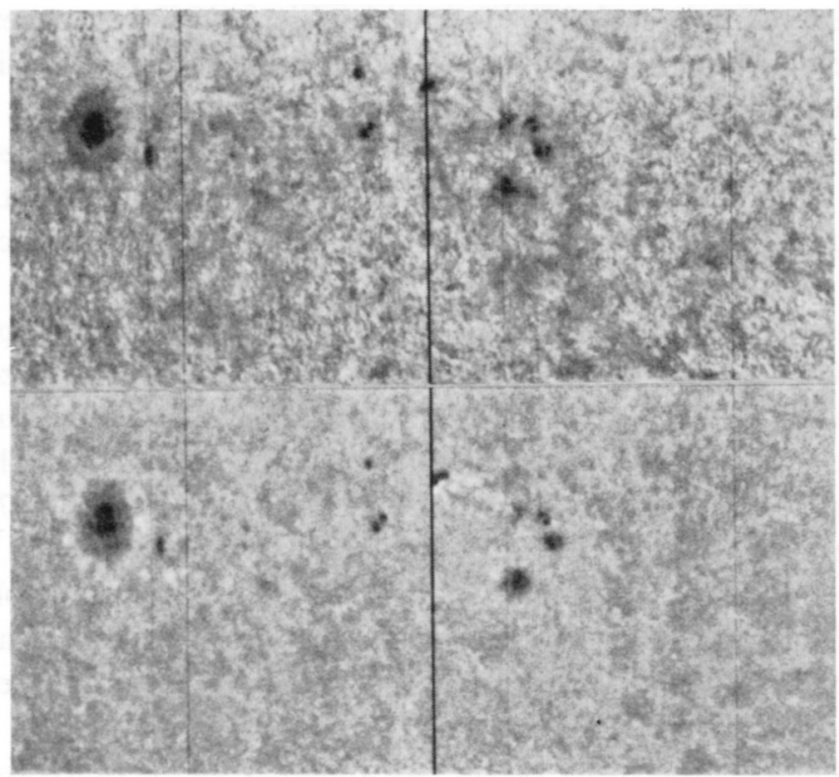

\section{Fe II}

$4924 R$

\section{$\mathrm{Fe}$ II}

\section{CORE}

\section{JULY 9, $1970 \quad 60,000 \mathrm{KM}$}

Fig. 3. Comparison of the appearance of spectroheliograms taken on the $\lambda 4924$ red wing and in the $\lambda 4924$ core respectively. 
on the line studied. A spatial resolution of $1 \mathrm{~s}$ was achieved for periods of $30 \mathrm{~min}$ to $1 \mathrm{hr}$ on about $25 \%$ of the mornings observed. Although the study of these movies is only beginning, a few of the most striking features will be listed in this paper. Here we shall describe some preliminary observations made using the $4924 \AA$ line of FeII.

Figure 1 compares simultaneous spectroheliograms made in the red wings of FeII $\lambda 4924$ and FeI $\lambda 4071$. Dark plages and network appear on the $\lambda 4924$ spectroheliogram and correspond roughly to the bright plages and network on the $\lambda 4071$ spectroheliogram. It is already known (see Figure 2 taken from Sheeley and Engvold, 1970) that this bright network in $\lambda 4071$ is cospatial with photospheric magnetic fields.

Figure 3 compares spectroheliograms made in the $\lambda 4924$ red wing and core respectively. Although these spectroheliograms were not taken simultaneously, they were taken within $30 \mathrm{~min}$ and the comparison is typically what one observes with simultaneous pictures. The core spectroheliogram shows active region brightenings characteristic of the middle chromosphere and a somewhat confusing diffuse brighterthan-average network similar to that seen in the cores of so many medium-strong lines such as Mgi $\lambda 5183$ (Chapman and Sheeley, 1968). However, in Figure 3 the dark network in FeII $\lambda 4924$ is not as striking as that of Figure 1. In particular, the network seems to be visible, not because it is much darker than average, but because the velocity granulation characteristic of nonmagnetic regions does not occur here. An examination of the data to determine the reason for this difference has not yet begun.

Figure 4 emphasizes the relation between the dark network in $\lambda 4924$, the magnetic field pattern, and the bright network of $\lambda 4071$. In this figure, the $\lambda 4071$ spectroheliogram was taken simultaneously with the $\lambda 4924$ red-wing spectroheliogram first in right-circularly-polarized light and $40 \mathrm{~s}$ later in left-circularly-polarized light. It is seen that the magnetic signal alternately reinforces and cancels the dark plages in the $\lambda 4924$ red wing depending on the magnetic polarity and the 'handedness' of the circular polarization.

Figure 5 shows how the $\lambda 4924$ line can be used to make a Zeeman photograph. Simultaneous red-wing $\lambda 4924$ spectroheliograms in right and left circularly-polarized light are shown together with the Zeeman photograph obtained by cancelling them. This figure represents one frame of a sequence taken every $30 \mathrm{~s}$ for one hour. This magnetic movie looked essentially the same as those seen in Ca I $\lambda 6103$ (Vrabec, 1971) and the brightness field in CN $\lambda 3883$ (Sheeley, 1969).

Figure 6 summarizes combinations of spectroheliograms taken on red and violet wings of $\lambda 4924$. Simultaneous violet-wing and red-wing $\lambda 4924$ spectroheliograms are shown together with the intensity field obtained by adding them and the velocity field obtained by subtracting them. Several features are clear:

(1) The dark network is much more visible on the red-wing than on the violet-wing spectroheliogram.

(2) The dark network appears on the 'sum' spectroheliogram in which doppler shifts have been cancelled. This indicates that the network is really darker than its surroundings and is not solely the result of doppler shifts.

(3) The region of the dark network appears on the velocity spectroheliogram and 


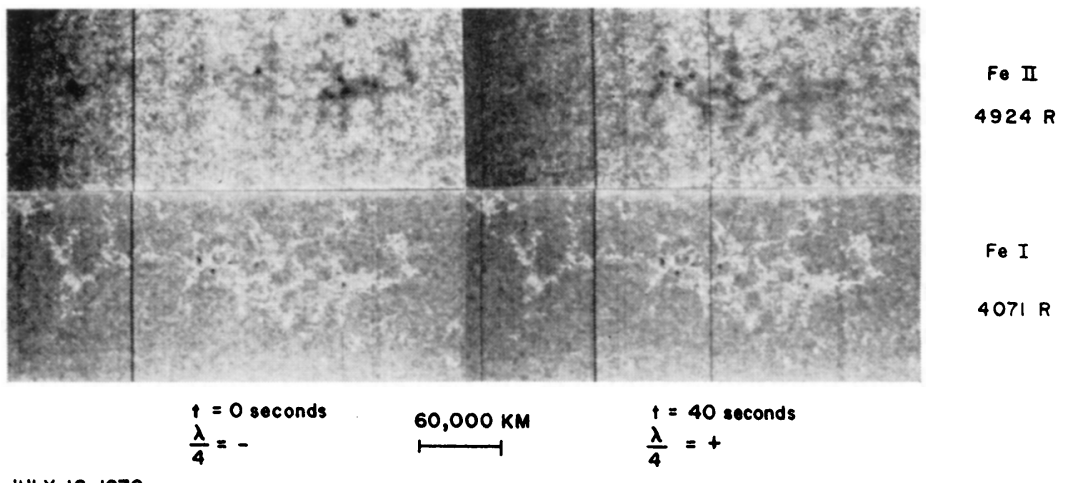

Fig. 4. Comparison between the $\lambda 4071$ bright network and the magnetically polarized $\lambda 4924$ dark network.
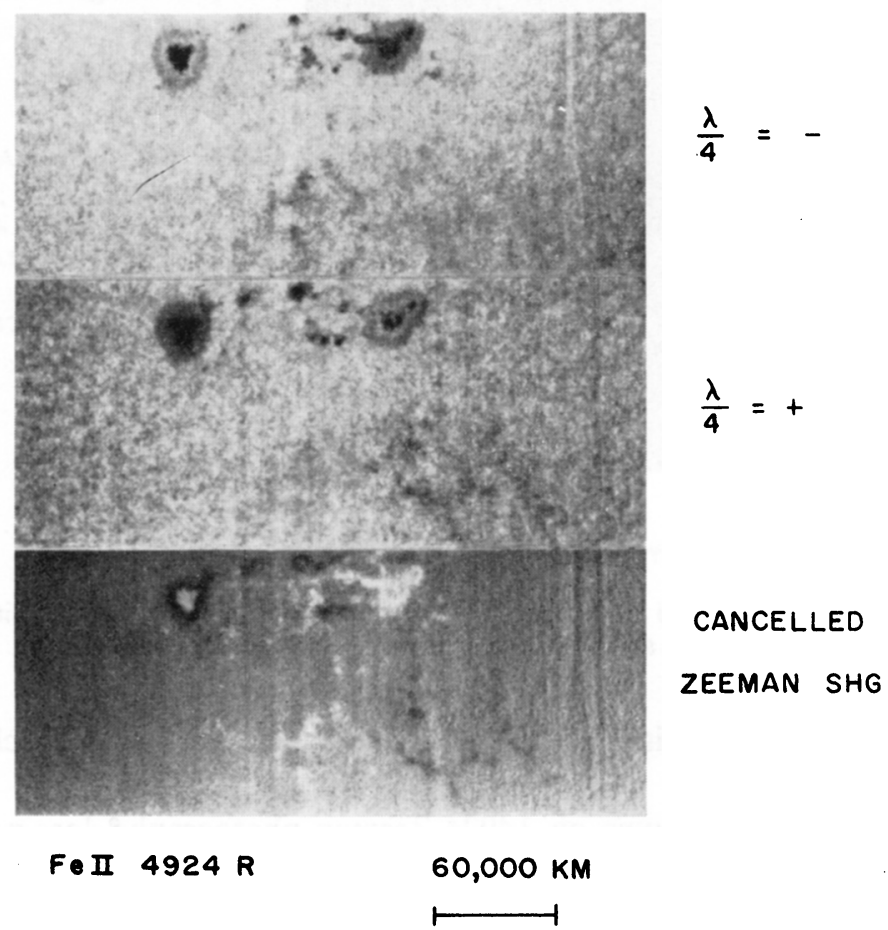

JULY 28, 1970

Fig. 5. Comparison between uncancelled and cancelled $\lambda 4924$ Zeeman spectroheliograms showing the appearance of the magnetic field as determined with this Fe Ir line. 
in this region the small-scale velocity is severely inhibited. Furthermore there is a definite downward direction to the velocity in the dark plage region.

A number of properties of the $\lambda 4924$ dark plage regions have been learned by viewing the movies:

(1) The visibility of the red-wing dark plages oscillates with a definite 5 min period.

(2) The visibility of the plages on the 'sum' spectroheliogram (obtained by adding the violet and red-wing spectroheliograms) does not oscillate. In particular, when frames in which a dark plage is no longer visible (due to its being $180^{\circ}$ out of phase

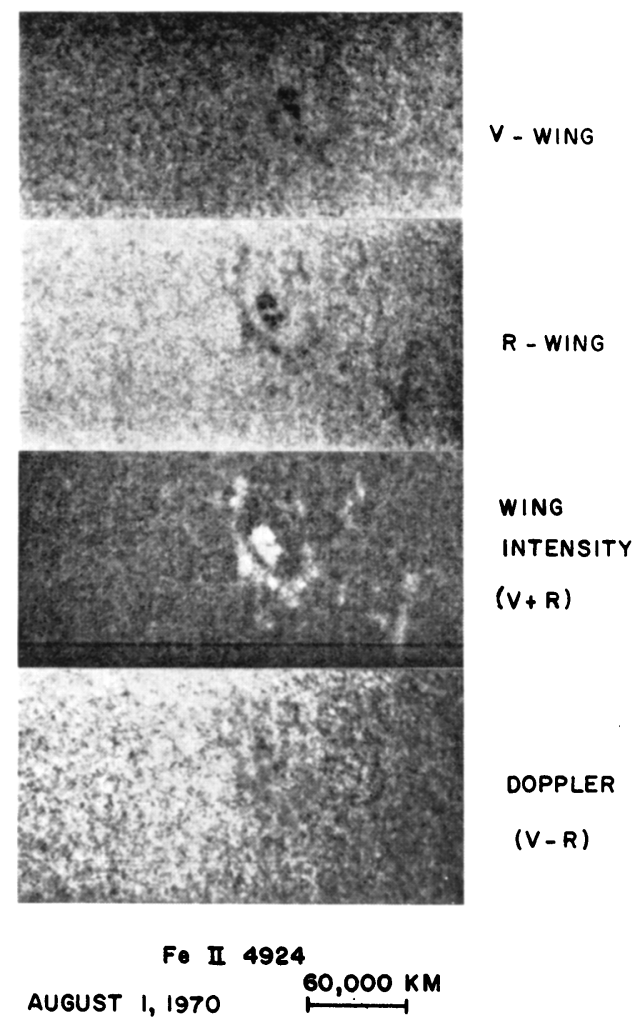

Fig. 6. Comparison between (a) violet wing; (b) red wing; (c) average wing intensity; (d) velocity field, as determined with the FeII $\lambda 4934$ line.

with its time of maximum visibility) are added, the dark plage appears clearly on the sum picture.

(3) The magnetic field shown on $\lambda 4924$ Zeeman photographs does not share this 5 min oscillation. Rather it shuffles about on the solar surface just the way the $\mathrm{CN}$ bright structures and the $\mathrm{Ca}$ I $\lambda 6103$ magnetic fields are known to move (see references above).

Further study of these movies as well as movies obtained in other lines listed above is presently underway. 
In summary, although the study of these movies is only beginning, a few of the most striking features are:

(1) On a short time scale, the 5 min oscillations of velocity and intensity are clearly visible.

(2) On a long time scale, the 15 min shuffling of brightness and magnetic fields are clearly visible. Surrounding sunspots, 'spheres of influence' have been observed in which the surface motions of the fields are predominantly outward from the spots.

(3) In magnetic regions the velocity field behaves differently than in non-magnetic regions. In field regions, the velocities are generally downward and the amplitude of the 5 min oscillation appears considerably reduced from the non-field amplitude.

(4) Dark plages, visible on the red wing of Fe II $\lambda 4924$, correspond spatially to the magnetic field pattern and show a striking oscillation of contrast with a 5 min period. Neither the magnetic fields nor the average wing intensity (obtained by adding $V$-wing and $R$-wing spectroheliograms) share in this oscillation.

\section{Acknowledgements}

The help and collaborations of A. Bhatnagar, C. C. Curtis, B. Gillespie, S.-Y. Liu, and R. A. Shine is gratefully acknowledged.

\section{References}

Chapman, G. A. and Sheeley, Jr., N. R.: 1968, Solar Phys. 5, 446.

Sheeley, Jr., N. R.: 1969, Solar Phys. 9, 347.

Sheeley, Jr., N. R. and Engvold, O.: 1969, 'Spectroheliograms in FeII $\lambda 4924$ ', paper presented at AAS Solar Meeting, Pasadena, Calif., Febr. 18-21, 1969.

Sheeley, Jr., N. R. and Engvold, O.: 1970, Solar Phys. $12,75$.

Vrabec, D.: 1971, this volume, p. 329. 